\title{
A review on natural therapy for seizure disorders
}

\begin{abstract}
Anti-seizure drugs are mainly used to treat epilepsy, but have been associated with certain side effects. Apart from drug therapy, other treatment options such as herbs, vitamins, biofeedback and acupuncture are available. However, these options are not widely used because the mechanism of such therapies is not fully understood. In the recent past, there has been an increasing interest among the public and scientist to explore the herbal treatments. Therefore, this review discusses the current status of natural therapies namely ketogenic diet, self-control method, acupuncture and chiropractic. The challenges and scope of applying these therapies as treatments for seizure disorders are further highlighted. Hence, this review provides a comparison of the current natural therapies, but particularly focuses on meditation and acupuncture for patients interested in such treatment options.
\end{abstract}

Volume 3 Issue 2 - 2015

\author{
Qian Ying Tan, Uday P Kundap, Yatinesh \\ Kumari, Mohd Farooq Shaikh \\ Jeffrey Cheah School of Medicine and Health Sciences, Monash \\ University, Malaysia
}

\author{
Correspondence: Mohd Farooq Shaikh, Jeffrey Cheah School \\ of Medicine and Health Sciences, \\ Monash University, Malaysia, Tel +60I428324I0, \\ Email farooq.shaikh@monash.edu
}

Received: November 8, 2015 | Published: December II, 2015

Keywords: epilepsy, conventional therapy, natural therapy, herbal treatments, self control methods

Abbreviations: WHO, worid health organisation; VNS, vagus nerve stimulation; DBS, deep brain stImulation; CAM, complementary and alternative medicine; EEG, electroencephalogram; Rcts, randomized controlled trials; AEDs, anti-epileptic drugs

\section{Introduction}

World Health Organisation (WHO) defined epilepsy as a chronic disorder categorized by recurring seizures with seizures being a brief lapse of attention or muscle jerks, to severe and prolonged convulsions. The seizures are triggered by excessive electrical discharges in the neurons that are often unexpected and brief. Epilepsy is the most common chronic brain disorder globally with approximately 50million people worldwide being affected and nearly $80 \%$ occurring in developing countries. ${ }^{1}$ Scientists believe that the 4 main causes of epilepsy are brain infection, head injury, brain tumour and stroke. However, epilepsy remains a puzzle as up to $60 \%$ of epileptic patients have unknown aetiology. ${ }^{2}$ Signs and symptoms of seizures varies and is dependent on the area of the brain that has been affected and how far the affected area spans. Some of the temporary symptoms include loss of awareness or consciousness, disturbances of movement, sensation such as vision, hearing and taste, mood, or other cognitive functions. ${ }^{2}$ According to WHO, it is possible to diagnose and treat most epilepsy patients at the preliminary stages without having to use sophisticated medical equipment. ${ }^{1}$

Anticonvulsant drugs are the conventional treatment used in epileptic patients. These antiseizure drugs often control and reduce the frequency of seizures but not really cure them completely. Studies have showed that a number of dietary modifications, nutritional supplements, and hormones are beneficial for some patients with epilepsy. ${ }^{3}$ The long term use of herbs introduced in traditional medicines confirms their value in drug discovery. Historical evidences showed that herbal therapies were used to treat convulsive seizures for centuries. Herbal medicine has always played a significant role in Iranian, Indian, Egypt \& Chines culture and civilization. Thousands ofyears of history and hundreds of books have placed by each traditional medicine among the oldest and richest alternative medicines. Ethnopharmacological research proved that medicinal plants have immense potential as anticonvulsant drugs and have been confirmed by scientific studies in experimental and clinical studies. ${ }^{4}$

\section{Conventional therapy}

Before diving directly into natural therapy, some aspects of conventional therapy are first discussed to understand the current treatment options available for people with epilepsy. Figure 1 shows the possible conventional treatment options available for epilepsy.

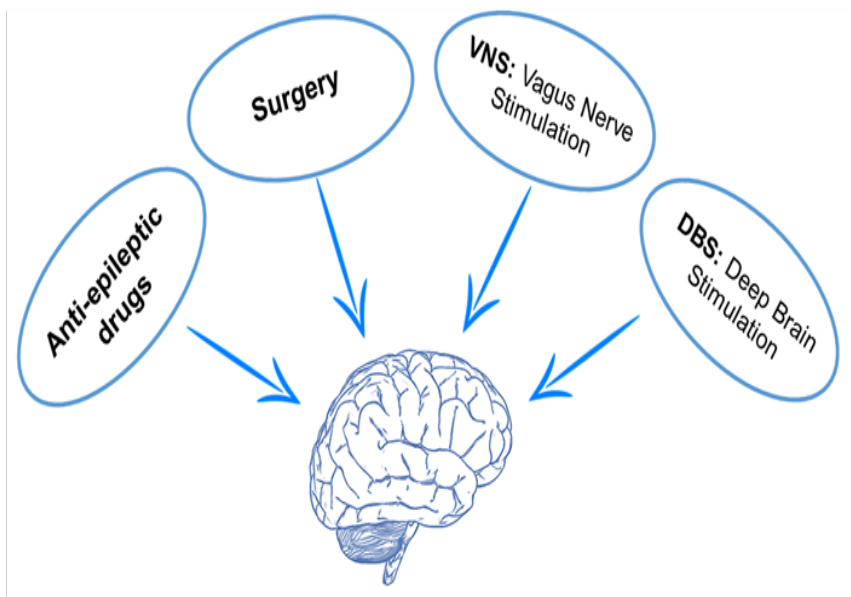

Epilepsy Treatment Options

Figure I Conventional epilepsy treatment options.

\section{Anti-epileptic drugs}

Anti-epileptic drugs (AEDs) discovery nearly doubled since the 1980s and are the key form of treatment option for epileptic patients. ${ }^{5,6}$ There are more than two dazone of AEDs used for the therapy and some of them are effective against a specific type of epilepsy. ${ }^{7}$ If the seizures are not controlled with a single AED, a combination of AEDs (polytherapy) is normally taken. ${ }^{8}$ Some AEDs added to first line AED are called second line AEDs. ${ }^{9}$ However, although AEDs prove to be the main treatment regime for epileptic patients, approximately $30 \%$ of epilepsy patients remain unresponsive towards drug treatment and continue to experience seizures whilst being on AEDs. ${ }^{10,11}$ One of the major issue with AEDs are chemophobia associated with them. Memory impairment, cognitive dysfunction, attention, problem 
solving ability, performance or visuomotor tasks; are greatly affected with barbiturates, hydantoins and carbamazepine.

\section{Surgery}

Surgery has been used for more than a century, with a dramatic increase of usage in the 1980s and 90s, reflecting its effectiveness as an alternative to seizure medicine. ${ }^{12}$ Epilepsy surgery is restricted to focal or localised type only. Some patients have showed improvement in memory and mood after surgery. ${ }^{13}$ A systematic review which included MEDLINE and Cochrane database found 55 articles, which includes two randomized clinical trials on 118 patients with temporal lobe epilepsy. A higher success rate of $58-70 \%$ was observed and patients found greater freedom from seizures. ${ }^{14}$ Compilcations associated with this type of surgery are memory problems, partial loss of sight, depression and other mood problems. ${ }^{15}$ However, postoperative outcomes are dependent on age of onset and prolonged duration of epilepsy or acute post-operative seizures. ${ }^{16}$ While the seizure outcomes are comparable to younger cohorts the cognitive risks of temporal lobe epilepsy surgery are greater for older patients, particularly those undergoing left temporal lobe resections. ${ }^{17} \mathrm{~A}$ Cochrane review further states that more diffused brain abnormalities associated with trauma to the brain or developmental abnormalities were not associated with a good outcome. ${ }^{18}$

\section{Vagus nerve stimulation and deep brain stimulation}

When AEDs and surgery is not the choice of therpay, neurostimulation may provide an alternative treatment. Vagus nerve stimulation (VNS) therapy involves stimulation of the left vagus nerve in the neck using a stimulator which sends mild electrical stimulations to the nerve that, in turn, sendstimulations to the brain.This stimulations helpsto calm down the irregular electrical brain activity whichgenerate electrical storm in the brain i.e, seizures. ${ }^{19}$ Usually, a device is implanted in the body which provide regular stimulations. Adverse effects coupled with implantation and stimulation generally include hoarseness, cough, dyspnea, pain, paresthesia, nausea and headache. ${ }^{20} \mathrm{~A}$ similar technique is deep brain stimulation (DBS) therapy essentially aims to reduce seizures frequency in epileptic patinets. In DBS therapy, electrodes are implanted into the specific brain areas and then stimuled with small regular electrical impulses. ${ }^{21}$ Adverse effects of some DBS include depression, subjective memory impairment, and possibly anxiety and confusion. ${ }^{22}$ It has been 25 years since the first VNS implantation in humans, yet researchers still have yet to fully comprehend the effects of neuro-stimulations on cognitive functioning. ${ }^{23}$

\section{Natural therapy}

Upon understanding the adverse effects and limitations surrounding conventional therapy, exploration of natural therapy is beneficial to aid in informed decision making when deciding on treatment options. Figure 2 illustrates the various natural therapies available for epileptic patients.

\section{Complementary and alternative medical therapies (CAM/Herbs)}

Substances from natural origin like plant, animal and minerals etc. have provided a continuing source of medicines since ancient times, and their use has been perpetuated for centuries in traditional medicine. ${ }^{24}$ Complementary and alternative medicine (CAM) has been used since a long time in various parts of the world and Western countries has gained interest over the last decades. In Western countries, natural products are used mainly to enhance general health and to prevent seizures. In some countries where they have a well developed traditional medical systems like traditional Chinese Medicine and Ayurveda, are depend on these options for treating epilepsy. A huge a lot of research all around the world is ongoing to decipher the scientific basis and mechanisms invloved. Both preclinical and clinical studies are taken up for the development of CAM in epilepsy. ${ }^{25,26}$

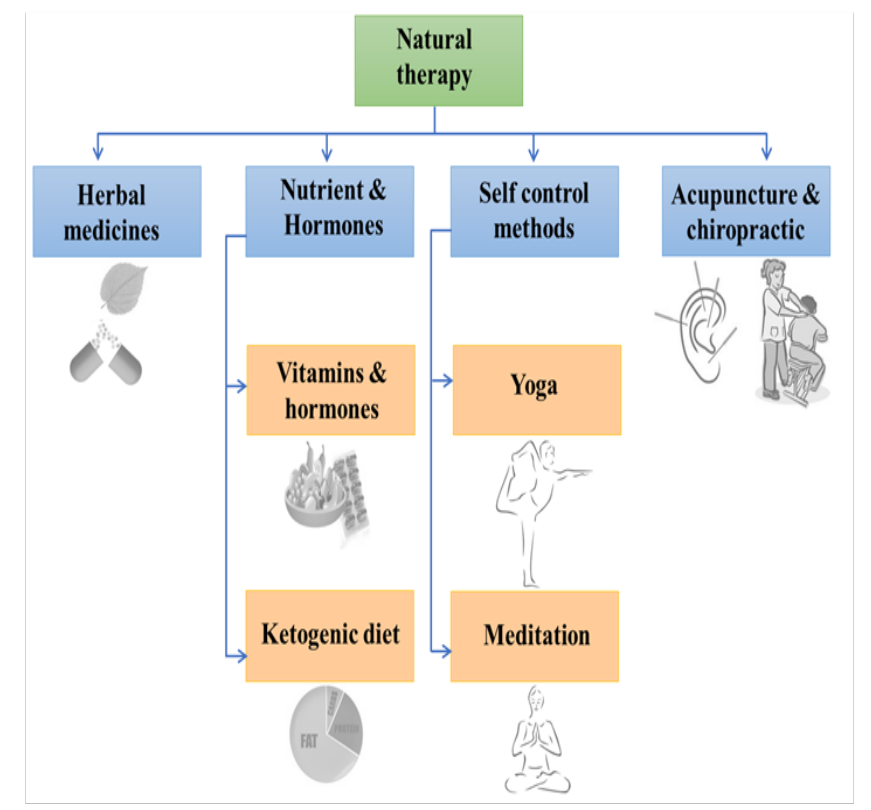

Figure 2 Natural epilepsy treatment options.

\section{Nutrient and hormones}

Nutritional therapy has been extensively studied and is seen as one of the more economical and promising options to treat epilepsy. ${ }^{27}$ Nutritional therapy includes vitamins, minerals, amino acids, hormones, herbs and ketogenic diets. Cochrane systematic reviews on nutritional therapy are limited but one concludes that there is no convincing evidence of vitamins improving seizure control or preventing side effects for people with epilepsy. ${ }^{28}$ According to Cochrane systematic reviews, there is also no current evidence sufficiently supporting the use of traditional Chinese herbs as treatment for epilepsy. ${ }^{29}$ However, Ketogenic diet has had substantial backing from the Cochrane database and is said to improve seizure control. ${ }^{30}$ The lack of clinical trials implores for more comprehensive studies to be carried out to provide evidence for nutritional therapy options such as vitamins, minerals and hormones to study their beneficial effects on epileptic patients.

\section{Self-control methods}

Meditation: Meditation has been practiced over centuries and is one of the most popular relaxation techniques. Some believe that transcendental meditation can be used as a form of epileptic treatment because the reduction of stress meant reduced incidence of seizures. ${ }^{31}$ One study specifically on epileptic patients has found that meditation reduced the frequency and severity of epileptic seizures. ${ }^{32}$ However, meditation has also been reported to be potentially dangerous for people with epilepsy. ${ }^{31}$ The practice of meditationis said to produce hormonal changes mimicking those of the inhibitory neurotransmitter c-aminobutyric acid (GABA). It has been hypothesized that meditation increases brain GABA by causing muscle relaxation, reducing insulin secretion, ketogenesis and subsequent ketosisenhanced entry of glutamate(the amino acid substrate of GABA) into 
synaptosomes. ${ }^{33}$ This allows for more glutamate to be converted to GABA. Such inhibitory neurotransmission induced by meditation might thus underlie suggestedantiepileptic effects. ${ }^{33}$ However, according to Jaseja, meditation may predispose individuals to epilepsy because of the increase in synchrony, glutamate and serotonin levels during meditation. ${ }^{34}$ Glutamate and serotonin are implicated in epileptogenesis as they are neuro-excitatory and may yield unfavourable outcomes when in high amounts as seen during meditation. ${ }^{34}$ Hence, it is essential to be well informed of the potential risks and benefits of relaxation techniques for people with epilepsy. ${ }^{31}$

Yoga: Yoga is another common relaxation technique that is said to induce relaxation and stress reduction. This influences the electroencephalogram and the autonomic nervous system, thus controlling seizures. ${ }^{35}$ Hence, the reduction in stress, increased PNS activity and increased brain GABA levels associated with yogabased interventions is said to be beneficial. ${ }^{36}$ It is said that yoga decreases seizure index and increase quality of life of epileptic patients. ${ }^{37}$ A Cochrane review showed evidence that supports this notion. It includedtwo trials in a sample of 50 people, with 18 who undertook yoga and 32 took AEDs served as control. Results revealed no statistically significant differences between the two groups. ${ }^{35,37}$ Furthermore, the evidence indicatesthat beneficial outcomes is limited and of low quality. ${ }^{35}$ Hence,no reliable conclusions can be drawn concerning the efficacy of yoga as a treatment for uncontrolled epilepsy due to the limited number of studies, limited participants randomized to yoga, lack of blinding and limited data on qualityof-life outcome. ${ }^{35}$ Yoga would be an attractive therapeutic option for epilepsy if proved effective and so further high-quality research is needed to fully evaluate the efficacy of yoga for epilepsy. In one study, the practice of yoga, a simple form of meditation, reduced seizures and EEG changes in people with epilepsy. The effect of meditation was attributed to a reduction in the level of stress as evidenced by changes in skin resistance and levels of blood lactate and urinary vanillylmandelic acid.

Neurofeedback: Neurofeedback is another alternative treatments in self control method in whichr esearchers have started to supplement medical regimens with behavioral treatments. It is a formof biofeedback therapy based on self-modulation of the electroencephalogram (EEG) by the subject. In neurofeedback, a specific EEG frequencies is believed to influence seizure control as well as behavioral parameters. ${ }^{38}$ The value of neurofeedback for human epilepsy was first established by Sterman and his colleagues in 1974. Sterman reviewed all of the literature up to 2000 and found that most of the studies on neurofeedback in epilepsy depicted positive outcome. In his meta-analysis, $82 \%$ of patients demonstrated more than $30-50 \%$ reduction in seizures. Approximately 5\% remained seizure free for up to a year. In recentyears, Walker and colleagues, have applied a different approach to normalize the EEG of a person with epilepsy by using qEEG as a guide for neurofeedback training in clinical settings. ${ }^{39}$ The general approach is to determine theabnormalities which are obvious and significant. Then, efforts are made to train those areas in order tonormalize them. This trainingexhibitedsubstantial seizure reductions but not in termination of seizures.Downtraining of gamma activity over the spike focus is an effective method of preventing seizure activity with neuroofeedback systems. ${ }^{40}$

\section{Acupuncture and chiropractic}

Acupuncture for the treatment of epilepsy dates back manyyears and is currently on the rise among epileptic patients with some reporting a reduction in seizure frequency as a result. ${ }^{41}$ Figure 3 illustrates the possible mechanism of auricular acupuncture for the treatment of epilepsy. According to Cheuk and Wong in a Cochrane review that included 17 randomized controlled trials (RCTs) with 1538 participants of many ageswho were suffering mainly from generalized epilepsy, acupuncture was said to possess no adverse effects towards epileptic patients. ${ }^{42}$ However, the included trials were small, heterogeneous and had a high risk of bias. It remains uncertain whether acupuncture is effective and safe for treating people with epilepsy. Hence, current evidence does not support acupuncture as a form of epilepsy treatment. ${ }^{42}$ In addition to that, another higher quality Norwegian trial documented no beneficial outcomes from acupuncture, with no improvement in the mean seizure frequency, seizure-free weeks, or quality of life in adults. ${ }^{41}$

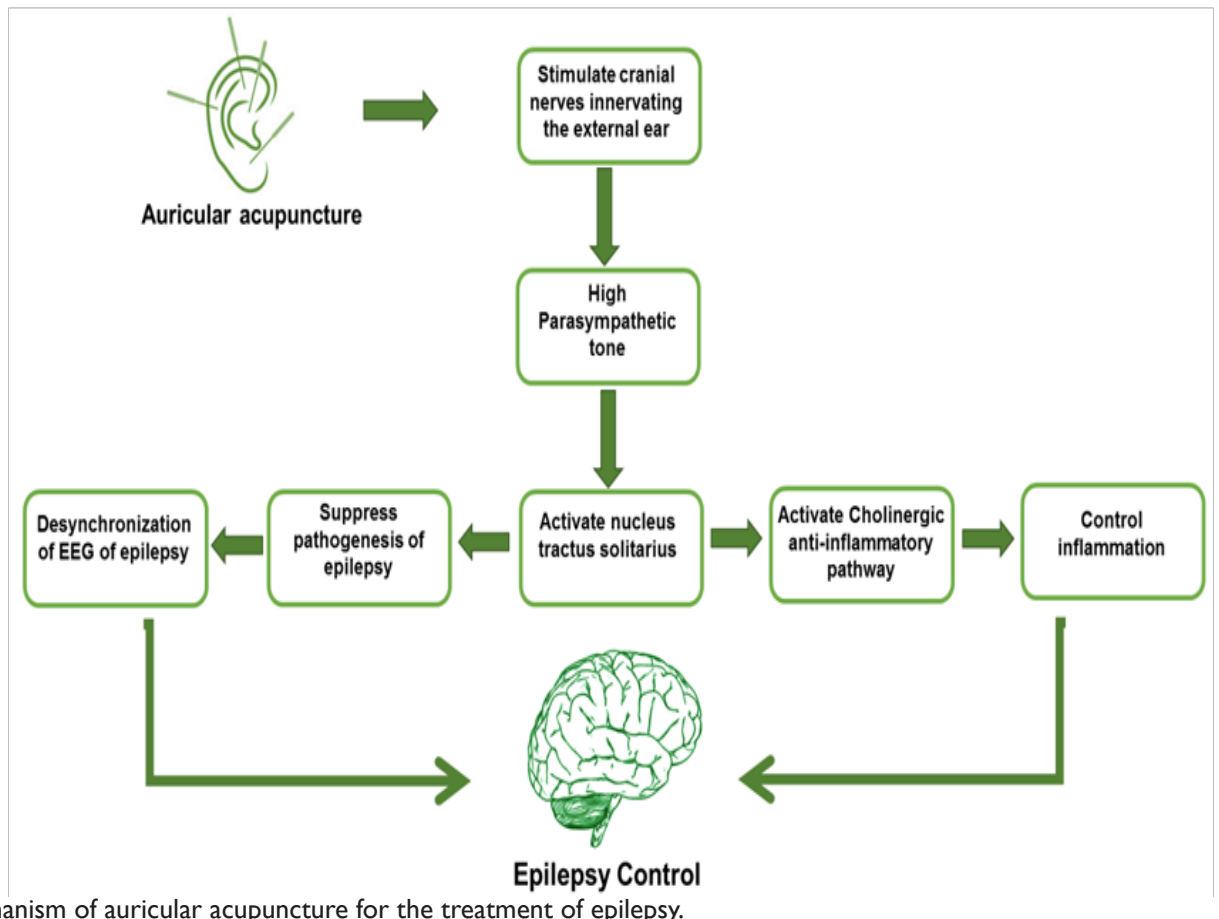

Figure 3 Probable mechanism of auricular acupuncture for the treatment of epilepsy. 
Chiropractic in epileptic patients is commonly the correction of the upper cervical area to amend vertebral subluxation. ${ }^{43}$ It is theorized that a reduction of abnormal nerve impulses to the brain can be attained through correcting vertebral subluxation in the upper cervical spine with professional chiropractic. ${ }^{43}$ Of the 17 cases that were studied, 15 showed promising results after undergoing chiropractic care ${ }^{43}$ However, such a theory is not backed by scientific mechanism, leaving the exact mechanism of which chiropractic care alleviates epilepsy symptoms unknown. Furthermore, there is no available Cochrane review that supports chiropractic care as a treatment option for epilepsy. Hence, even though chiropractic care has been advocated to be beneficial, there is a need for more investigations and clinical trials to be carried out in order to validate its' effectiveness.

\section{Discussion and conclusion}

Extensive reviews and researches have been done on the nutritional aspect of natural therapy. Hence, this article focuses on literature related to self-control methods (meditation and yoga), acupuncture and chiropractic as therapy for seizure disorders due to the lack of literature reviews of the aforementioned therapies. Meditation, yoga, acupuncture and chiropractic practices show promising outcomes for epileptic patients but has to be extensively studied to provide substantial evidence of their beneficial effects. (Table 1) summarizes the findings of various review articles to indicate the strength of current evidences supporting meditation, yoga, acupuncture and chiropractic as epilepsy treatment therapies.

There is no particularprocedure to halt a seizure, and there is no assurance about its effectiveness every time and for everyone. Meditation and yoga as epilepsy treatments still remain a debate with plenty of scope for researchers to explore. Acupuncture and chiropractic are sometimes considered substitutes to conventional medicine as they may change brain activities to reduce seizures. Theoretically, both of these practicessounds virtuous but there is lack of scientific evidence to support them as an effective in epilepsy management. Various dietary modifications may also help in decreasing seizures, ketogenic diet is among one of them. This high fat (Ketogenic) diet is found to be effective in children, but the restrictions are challenging. In conclusion, the brain is a complex network and each and every case is different. Hence, natural treatments may complement existing medical treatment. However, there is insufficient evidence to assess either the benefits or adverse effects of natural therapy and further studies are needed.

Table I Comparison of meditation, yoga, acupuncture and chiropractic as natural therapies for epilepsy

\begin{tabular}{|c|c|c|}
\hline $\begin{array}{l}\text { Natural } \\
\text { therapy }\end{array}$ & $\begin{array}{l}\text { Cochrane } \\
\text { review }\end{array}$ & Evidence \\
\hline Meditation & Absent & 2 case studies \\
\hline Yoga & Present & 2 unblinded trials with 50 participants \\
\hline Acupuncture & Present & $\begin{array}{l}\text { I7 randomised controlled trials with } \\
\text { I538 participants }\end{array}$ \\
\hline Chiropractic & Absent & $\begin{array}{l}\text { I } 7 \text { individual reports, } 15 \text { got treated, } \\
\text { but no scientific evidence is available }\end{array}$ \\
\hline $\begin{array}{l}\text { Neuro } \\
\text { feedback }\end{array}$ & Absent & $\begin{array}{l}10 \text { individual studies, total } 87 \\
\text { participants. There was no evidence } \\
\text { of significant statistical heterogeneity. }\end{array}$ \\
\hline
\end{tabular}

\section{Acknowledgements}

This work is supported by the eScience Fund of Ministry of Science, Technology and Innovation (MOSTI), Malaysia (Grant No. 0602-10-SF0250).

\section{Conflict of interest}

Author declares that there is no conflict of interest.

\section{References}

1. Sharma S, Dixit V. Epilepsy-A Comprehensive Review. International Journal of Pharma Research \& Review. 2013;2(12):61-80.

2. Hirak K, Mukhopadhyay, Chandi CK, et al. Epilepsy and its Management: A Review. Journal of Pharma Sci Tech. 2012;1(2):20-26.

Limitation

The available studies are limited, reviews provide

contrasting views and mechanism not fully 32-34 understood

The available studies are limited with limited number of participants randomized to yoga, lack of $\quad 35,37$ blinding and limited outcome measures

\section{Available RCTs is small, heterogeneous and have a 42} high risk of bias

The available studies are limited and there is a lack of understanding of the mechanism of chiropractic as a therapy

The unclear quality of included studies, the small sample sizes and the possibility of publication bias. 4
References

3. Malvi RK, Bigoniya P, Sethi S, et al. Medicinal plants used in the treatment of epilepsy. IRJP. 2011;2(2):32-39.

4. Abdollahi FM, Shojaii A. Efficacy of Iranian traditional medicine in the treatment of epilepsy. BioMed research international. 2013;2013:1-8.

5. Brodie MJ. Antiepileptic drug therapy the story so far. European Journal of Epilepsy. 2010;19(10):650-655.

6. Walia KS, Khan EA, Ko DH, et al. Side effects of antiepileptics-a review. Pain practice. 2004;4(3):194-203.

7. Lagae L. Cognitive side effects of anti-epileptic drugs: the relevance in childhood epilepsy. Seizure. 2006;15(4):235-241.

8. Vining EP. Cognitive dysfunction associated with antiepileptic drug therapy. Epilepsia. 1987;28(S2):S18-S22. 
9. Perucca P, Meador KJ. Adverse effects of antiepileptic drugs. Acta Neurol Scand. 2005;Suppl 181:30-35.

10. Inoyama K, Meador KJ. Cognitive Outcomes of Prenatal Antiepileptic Drug Exposure. Epilepsy Research. 2015;114:89-97.

11. Beavis J, Kerr M, Marson AG. Non-pharmacological interventions for epilepsy in people with intellectual disabilities. Cochrane Database of Systematic Reviews. 2007;(4):CD005502.

12. Kemeny AA. Surgery for epilepsy, Seizure. 2001.

13. Guldvog B, Loyning Y, Hauglie HE, et al. Predictive factors for success in surgical treatment for partial epilepsy: a multivariate analysis. Epilepsia. 1994;35(3):566-578.

14. Cascino GD. Surgical treatment for epilepsy. Epilepsy research 2004;60(2):179-186.

15. Kelly KM, Chung SS. Surgical treatment for refractory epilepsy: review of patient evaluation and surgical options. Epilepsy research and treatment. 2011;2011:1-10.

16. Jayalakshmi S. Outcome of surgery for temporal lobe epilepsy in adults A-cohort study. International Journal of Surgery. 2015.

17. Thompson PJ, Baxendalea SA, McEvoya AW, et al. Cognitive outcomes of temporal lobe epilepsy surgery in older patients. Seizure - European Journal of Epilepsy. 2015;29:41-45.

18. West S, Nolan SJ, Cotton J, et al. Surgery for epilepsy. Cochrane Database of Systematic Reviews. 2015.

19. Sen A, Selway R, Nashef L, Vagus nerve stimulation.

20. Privitera MD, Welty TE, Ficker DM, et al. Vagus nerve stimulation for partial seizures. Cochrane Database of Systematic Reviews. 2002;(1):CD002896.

21. Mehanna R, Lai EC. Deep brain stimulation in Parkinson's disease. Transl Neurodegener. 2013;2(1):22.

22. Sprengers M, Vonck K, Carrette E, et al. Deep brain and cortical stimulation for epilepsy. Cochrane Database Syst Rev. 2017;7:CD008497.

23. Vonck K, Raedt R, Naulaerts J, et al. Vagus nerve stimulation...25years later! What do we know about the effects on cognition? Neurosci Biobehav Rev. 2014;45:63-71.

24. Samleti A, Sharma N, RD Tambole, et al. Traditional herbs used in treatment of epileptic seizures. International Journal of Pharmaceutical and Chemical Sceinces. 2012;1(3):1062-1068.

25. Tripathi M. Ayurvedic medicine and epilepsy. Neurological Journal of Southeast Asia. 2000;5:1-4.

26. Ekstein D, Steven CS. Natural Products in Epilepsy-the Present Situation and Perspectives for the Future. Pharmaceuticals. 2010;3(5):1426-1445.

27. Sancheti J, Shaik MF, Akhade M, et al. Nutritional therapy for epilepsy. J Pharm Bio Sci. 2013;4(2013):149-156.
28. Narasimhan RL, Sridharan R. Vitamins for epilepsy. Cochrane Database of Systematic Reviews. 2005

29. Qifu Li, XC, Li He, et al. Traditional Chinese medicine for epilepsy. Cochrane Database Syst Rev. 2009;3:CD006454.

30. Levy RG, Cooper PN, Giri P. Ketogenic diet and other dietary treatments for epilepsy. Cochrane Database Syst Rev. 2012;3:CD001903

31. Bellon M. Relaxation techniques for people with epilepsy. The Epilepsy Report. 2007.

32. Chalmers R. Transcendental meditation does not predispose to epilepsy Medical Hypotheses. 2005;65(3):624-625.

33. Lansky EP, St. Louis EK. Transcendental meditation: A double-edged sword in epilepsy? Epilepsy Behav. 2006;9(3):394-400.

34. Jaseja H. Meditation potentially capable of increasing susceptibility to epilepsy - A follow-up hypothesis. Medical Hypotheses 2006;66(5):925-928.

35. Mariangela P, Kalpana S, Sridharan R. Yoga for epilepsy. Cochrane Database of Systematic Rev. 2015.

36. Streeter CC, Gerbarg PL, Saper RB, et al. Effects of yoga on the autonomic nervous system, gamma-aminobutyric-acid, and allostasis in epilepsy, depression, and post-traumatic stress disorder. Medical Hypotheses. 2012;78(5):571-579.

37. Lundgren T, Dahl J, Yardi N, et al. Acceptance and Commitment Therapy and yoga for drug-refractory epilepsy: A randomized controlled trial. Epilepsy Behav. 2008;13(1):102-108.

38. Monderer RS, Harrison DM, Haut SR. Neurofeedback and epilepsy. Epilepsy Behav. 2002;3(3):214-218

39. Walker JE, Kozlowski GP. Neurofeedback treatment of epilepsy. Child and adolescent psychiatric clinics of N Am. 2005;14(1):163-176.

40. Kotchoubey B, Strehla U, Holzapfel S, et al. Negative potential shifts and the prediction of the outcome of neurofeedback therapy in epilepsy. Clin Neurophysiol. 1999;110(4):683-686.

41. Kloster R, Larsson PG, Lossius R, et al. The effect of acupuncture in chronic intractable epilepsy. Seizure. 1999;8(3):170-174.

42. Cheuk DK, Wong V. Acupuncture for epilepsy. Cochrane Database of Systematic Rev. 2008;4:CD005062.

43. Pistolese RA. Epilepsy and seizure disorders: A review of literature relative to chiropractic care of children. Journal of Manipulative and Physiological Therapeutics. 2001;24(3):199-205.

44. Tan G, Thornby J, Hammond DC, et al. Meta-analysis of EEG biofeedback in treating epilepsy. Clinical EEG and Neuroscience. 2009;40(3):173-179. 